\title{
Bit Loading for MIMO with Statistical Channel Information at the Transmitter and ZF Receivers
}

\author{
Ana García Armada $\dagger$, Li Hong $\dagger$ and Angel Lozano $\ddagger$ \\ $\dagger$ Univ. Carlos III de Madrid, 28911 Leganés, Spain \\ $\ddagger$ Universitat Pompeu Fabra, 08005 Barcelona, Spain
}

\begin{abstract}
For single-user MIMO communication with uncoded and coded QAM signals, we propose bit and power loading schemes that rely only on channel distribution information at the transmitter. To that end, we develop the relationship between the average bit error probability at the output of a $\mathrm{ZF}$ linear receiver and the bit rates and powers allocated at the transmitter. This relationship, and the fact that a $\mathrm{ZF}$ receiver decouples the MIMO parallel channels, allow leveraging bit loading algorithms already existing in the literature. We solve dual bit rate maximization and power minimization problems and present performance results that illustrate the gains of the proposed scheme with respect to a non-optimized transmission.
\end{abstract}

\section{INTRODUCTION}

Multiple-input multiple-output (MIMO) is quickly becoming a central ingredient in the design of wireless systems. In MIMO communication, the adaptation of the modulation and coding at the transmitter side according to the channel characteristics allows reducing the transmission power and/or enhancing the data rates. However, it is not always feasible to have instantaneous knowledge of the channel at the transmitter. We focus here on the case that the receiver has (perfect) instantaneous Channel State Information (CSIR) but the transmitter has only access to its distribution (CDIT). This is a practical case that applies, particularly, to situations where the channel varies rapidly. Under CDIT, the input cannot be adapted to the instantaneous state of the channel and thus SVD (singular value decomposition) cannot be used to diagonalize the channel. Achieving capacity requires a complex Gaussian input vector with a covariance that depends on the channel distribution [1].

In practice, however, discrete constellations are used in lieu of Gaussian signals. Determining the optimum signalling strategy with discrete constellations is difficult in general, and thus we take the pragmatic approach of using the spatial signalling directions indicated by the capacity-achieving covariance. Bit and power loading algorithms [2] are available for parallelchannel settings, e.g., OFDM. Our goal is to devise a bit and power loading scheme for MIMO that can operate on the basis of CDIT only. For practical reasons, we consider only linear receivers. In particular, we use a zero-forcing (ZF) receiver rather than an MMSE (minimum mean squared error) receiver because with $\mathrm{ZF}$ we can-at the expense of some noise enhancement-recover independent parallel signals at

This work has been partly funded by projects MULTI-ADAPTIVE (TEC2008-06327-C03-02) and COMONSENS (CSD2008-00010). the receiver. The fact that these parallel signals do not mutually interfere is the key to leveraging bit loading algorithms that have traditionally been used for OFDM. We shall try to address the following questions:

- What bit rates can be achieved, with discrete constellations using the transmit directions given by the capacityachieving input covariance, at a certain average bit error probability (BER)?

- What is the optimum power loading?

- How much can be gained in terms of signal-to-noise ratio relative to a non-optimized transmission?

In order to answer these questions, it is essential to establish a relationship between the average BER after the ZF receiver and the powers allocated at the transmitter under the premise of CDIT.

\section{SySTEM MODEL}

Let $n_{\mathrm{T}}$ and $n_{\mathrm{R}}$ denote, respectively, the number of transmit and receive antennas. The input is the $n_{\mathrm{T}} \times 1$ complex vector

$$
\mathbf{x}=\sqrt{\mathbf{P}} \mathbf{V s}
$$

where $\mathbf{s}$ is a vector of unit-energy $M$-QAM ( $M$-ary Quadrature Amplitude Modulation) data. The optimization of the constellation cardinality for each entry of $\mathbf{s}$ is one of the goals of this paper. The unitary matrix $\mathbf{V}$ is a linear precoder whose columns define the signalling vectors while the diagonal matrix $\mathbf{P}$ determines the fraction of the available power allocated to each such vector. The $n_{\mathrm{R}} \times 1$ received vector is

$$
\mathbf{y}=\mathbf{H x}+\mathbf{n}
$$

where the $n_{\mathrm{R}} \times n_{\mathrm{T}}$ channel matrix $\mathbf{H}$ has correlation at the transmit side only such that

$$
\mathbf{H}=\mathbf{H}_{\mathbf{w}} \sqrt{\mathbf{R}_{\mathbf{t}}}
$$

where $\mathbf{H}_{\mathbf{w}}$ has i.i.d. circularly symmetric complex Gaussian entries with zero mean and unit variance while $\mathbf{R}_{\mathbf{t}}$ is the transmit correlation matrix. Finally, $\mathbf{n}$ is a zero-mean circularly symmetric complex Gaussian $n_{\mathrm{R}} \times 1$ noise vector. We define the average signal-to-noise ratio

$$
\mathrm{SNR}=n_{\mathrm{R}} \frac{E\left\{\mathbf{x}^{H} \mathbf{x}\right\}}{E\left\{\mathbf{n}^{H} \mathbf{n}\right\}}
$$

and make use of the normalization

$$
E\left\{\operatorname{Tr}\left[\mathbf{x x}^{H}\right]\right\}=n_{\mathrm{T}}=\operatorname{Tr}[\mathbf{P}]
$$


with $\operatorname{Tr}[\cdot]$ denoting the trace of a matrix. We also define the input signal covariance as

$$
\boldsymbol{\Phi}=E\left\{\mathbf{x x}^{H}\right\}=\mathbf{V P} \mathbf{V}^{H}
$$

For a rather general class of channel models [1], the capacity-achieving precoder $\mathbf{V}$ under CDIT contains the eigenvectors of $E\left\{\mathbf{H}^{H} \mathbf{H}\right\}$. This precoder is also shown to be optimal from an SINR standpoint with a linear MMSE receiver [7]. We shall use it as a pragmatic solution and verify its goodness via simulation. Using this precoder, we can then try to determine the optimum power allocation $\mathbf{P}$.

The $\mathrm{ZF}$ receiver essentially inverts the channel yielding at its output $\mathbf{z}=\mathbf{G y}$ with

$$
\mathbf{G}=(\mathbf{H} \sqrt{\mathbf{P}} \mathbf{V})^{\dagger}
$$

where $(\cdot)^{\dagger}$ denotes the Moore-Penrose pseudoinverse [3].

The SINR (Signal-to-Interference-plus-Noise Ratio) experienced by the signal transmitted on the $n$th signalling vector at the output of the $\mathrm{ZF}$ receiver is

$$
\mathrm{SINR}_{n}=\frac{\mathrm{SNR} / n_{\mathrm{T}}}{\left[(\mathbf{H} \sqrt{\mathbf{P}} \mathbf{V})^{H}(\mathbf{H} \sqrt{\mathbf{P}} \mathbf{V})\right]_{n, n}^{-1}}
$$

where $[\cdot]_{n, n}$ indicates the $(n, n)$ th entry of a matrix.

For the performance evaluations in Section $\mathrm{V}$, we will use the following transmit covariance matrix corresponding to suburban/rural environments with small angular spreads at the transmitter [6]

$$
\mathbf{R}_{\mathbf{t}}=\left[\begin{array}{llll}
1 & \rho & \rho^{4} & \rho^{9} \\
\rho & 1 & \rho & \rho^{4} \\
\rho^{4} & \rho & 1 & \rho \\
\rho^{9} & \rho^{4} & \rho & 1
\end{array}\right]
$$

\section{Average Bit Error Probability}

We are interested in the SINR statistics as a function of the input and the channel characteristics. The ultimate goal is to relate the average BER at the receiver (through the corresponding SINR) with the power allocation matrix $\mathbf{P}$. The output SINR for a ZF receiver in (8) on a transmit-correlated Rayleigh-faded channel with unequal powers, $n_{\mathrm{T}} \leq n_{\mathrm{R}}$, is characterized in [4]. It follows the Gamma distribution

$$
f_{\operatorname{SINR}_{n}}(s)=\frac{s^{\alpha-1} e^{-s / \theta}}{\Gamma(\alpha) \theta^{\alpha}}
$$

with parameters

$$
\begin{aligned}
\alpha & =n_{\mathrm{R}}-n_{\mathrm{T}}+1 \\
\theta & =\frac{1 / n_{\mathrm{R}}}{\left[\mathbf{R}^{-\mathbf{1}}\right]_{n, n}}
\end{aligned}
$$

where

$$
\mathbf{R}=\operatorname{SNR} \frac{n_{\mathrm{R}}}{n_{\mathrm{T}}} \sqrt{\mathbf{P}} \mathbf{V} \mathbf{R}_{\mathbf{t}} \mathbf{V}^{H} \sqrt{\mathbf{P}}
$$

Then, the average BER for the $n$th signal (the subindex $n$ is dropped for notational simplicity) can be obtained from the Gamma parameters as

$$
\bar{P}_{e}(\alpha, \theta)=\int_{0}^{\infty} P_{e}(s) \frac{s^{\alpha-1} e^{-s / \theta}}{\Gamma(\alpha) \theta^{\alpha}} d s
$$

where $\Gamma(\cdot)$ is the complete Gamma function and $P_{e}(\cdot)$ is the instantaneous BER function for the corresponding scalar signal, which depends on the modulation and coding scheme used at the transmitter. Expressions for $P_{e}(\cdot)$ and $\bar{P}_{e}(\cdot, \cdot)$ are developed in the following subsections for uncoded and coded $M$-QAM modulations.

\section{A. Uncoded M-QAM Modulations}

$P_{e}(\cdot)$ for uncoded $M$-QAM, conditioned on the fading realization, is given by the expressions [5] in Table I, first column, with

$$
Q(x)=\int_{x}^{\infty} \frac{e^{-u^{2} / 2}}{\sqrt{2 \pi}} d u .
$$

Table I

EXACT AND APPROXIMATE BER IN AWGN

\begin{tabular}{|c|c|c|}
\hline & BER & Approximate BER \\
\hline 2-PAM & $P_{e}(s)=Q(\sqrt{2 s})$ & $P_{e}(s) \approx \frac{1}{2 \sqrt{\pi}} e^{-s}$ \\
\hline 4-QAM & $P_{e}(s)=Q(\sqrt{s})$ & $P_{e}(s) \approx \frac{1}{2 \sqrt{\pi}} e^{-s / 2}$ \\
\hline$M$-QAM & $P_{e}(s) \approx \frac{4 Q\left(\sqrt{\frac{3 s}{M-1}}\right)}{\log _{2} M}$ & $P_{e}(s) \approx \frac{1}{\log _{2} M} \frac{2}{\sqrt{\pi}} e^{\frac{-3 s}{2(M-1)}}$ \\
\hline
\end{tabular}

At high SINR, we can further use

$$
Q(x) \approx \frac{1}{2 \sqrt{\pi}} e^{-\frac{x^{2}}{2}}
$$

to obtain the approximate BER expressions in Table I, second column. Plugging these approximate expressions into (14) and integrating, approximate average BER expressions as function of the Gamma parameters $(\alpha, \theta)$ for the uncoded case are found and presented in Table II. Their counterparts, obtained by integrating (14) with the formulas in the first column of Table I, can be expressed by means of the hypergeometric function

$$
{ }_{2} F_{1}(a, b ; c ; d)=\frac{\Gamma(c)}{\Gamma(b) \Gamma(c-b)} \int_{0}^{1} \frac{t^{b-1}(1-t)^{c-b-1}}{(1-t d)^{a}} d t
$$

as follows. For 2-PAM,

$\bar{P}_{e}=\frac{1}{2 \Gamma(\alpha)}\left[\Gamma(\alpha)-2 \sqrt{\frac{\theta}{\pi}} \Gamma\left(\frac{1}{2}+\alpha\right){ }_{2} F_{1}\left(\frac{1}{2}, \frac{1}{2}+\alpha ; \frac{3}{2} ;-\theta\right)\right]$

while, for 4-QAM,

$\bar{P}_{e}=\frac{1}{2 \Gamma(\alpha)}\left[\Gamma(\alpha)-\sqrt{\frac{2 \theta}{\pi}} \Gamma\left(\frac{1}{2}+\alpha\right){ }_{2} F_{1}\left(\frac{1}{2}, \frac{1}{2}+\alpha ; \frac{3}{2} ;-\frac{\theta}{2}\right)\right]$

and, for $M$-QAM,

$\bar{P}_{e} \approx \frac{\Gamma(\alpha)-\sqrt{\frac{6 \theta}{\pi(M-1)}} \Gamma\left(\frac{1}{2}+\alpha\right){ }_{2} F_{1}\left(\frac{1}{2}, \frac{1}{2}+\alpha ; \frac{3}{2} ; \frac{-3 \theta}{2(M-1)}\right)}{1 / 2 \log _{2}(M) \Gamma(\alpha)}$

Fig. 1 shows the excellent agreement between the expressions in Table II and those in (18), (19) and (20), for the operational range of interest $\left(\bar{P}_{e}<10^{-1}\right)$. On account of this agreement, we shall henceforth use the much simpler expressions in Table II. 
Table II

Approximate Average BER for Uncoded Modulations

\begin{tabular}{|c|c|}
\hline 2-PAM & $\bar{P}_{e} \approx \frac{1}{2 \sqrt{\pi}}(1+\theta)^{-\alpha}$ \\
\hline 4-QAM & $\bar{P}_{e} \approx \frac{1}{2 \sqrt{\pi}}\left(1+\frac{\theta}{2}\right)^{-\alpha}$ \\
\hline$M$-QAM & $\bar{P}_{e} \approx \frac{1}{\log _{2} M} \frac{2}{\sqrt{\pi}}\left(1+\frac{3 \theta}{2(M-1)}\right)^{-\alpha}$ \\
\hline
\end{tabular}

\section{B. Coded M-QAM Modulations}

Consider now $M$-QAM modulation in conjunction with a convolutional code of rate $r=k / n$ and minimum distance $d_{\text {free }}$ With hard-decision decoding, maximum likelihood reduces to a minimum distance criterion and the BER at the output of the decoder is upper bounded by [5]

$$
P_{c} \leq \frac{1}{k} \sum_{d=d_{\text {free }}}^{\infty} A_{d}\left(2 \sqrt{P_{e}\left(1-P_{e}\right)}\right)^{d}
$$

with $P_{e}$ the bit error probability of the corresponding uncoded $M$-QAM. In turn, $A_{d}$ is the number of paths in the trellis with distance $d$ from the all-zero path that merge with this all-zero path for the first time.

At high SINR, the summation in (21) is dominated by the first term and, if $P_{e}$ is small, we can further approximate

$$
P_{c} \approx \frac{1}{k} A_{d_{\text {free }}} 2^{d_{\text {free }}} P_{e}^{d_{\text {free }} / 2}
$$

Using the parameter

$$
\beta=\frac{A_{d_{\text {free }}}}{k} 2^{d_{\text {free }} / 2} \pi^{-d_{\text {free }} / 4}
$$

and the approximate $P_{e}$ expressions in Table I, second column, we can obtain by integrating (14) the average BER expressions in Table III.

Table III

AVERAGE BER FOR CODED MODULATIONS

\begin{tabular}{|c|c|}
\hline 2-PAM & $\bar{P}_{e}=\beta\left(1+\frac{d_{\text {free }}}{2} \theta\right)^{-\alpha}$ \\
\hline 4-QAM & $\bar{P}_{e}=\beta\left(1+\frac{d_{\text {free }}}{4} \theta\right)^{-\alpha}$ \\
\hline$M$-QAM & $\bar{P}_{e}=\frac{4}{\log _{2} M} \beta\left(1+\frac{3 d_{\text {free }}}{4(M-1)} \theta\right)^{-\alpha}$ \\
\hline
\end{tabular}

\section{Optimization PRoblems}

Denote by $M_{n}$ the cardinality of the modulation applied to the $n$th eigenvector of $\mathbf{V}$, by $\bar{P}_{e, n}$ its average BER after the $\mathrm{ZF}$ receiver, and by $\overline{\mathrm{BER}}$ the target average BER. Once the relationship between average BER and the parameters $(\alpha, \theta)$ has been established and the SINR can be expressed as function of the channel correlations and the power allocation, we are able to solve the following dual optimization problems.

\section{A. Maximize Bit Rate}

$$
\begin{gathered}
\max R=\sum_{n=1}^{n_{\mathrm{T}}} \log _{2} M_{n} \\
\text { s.t. } \bar{P}_{e, n} \leq \overline{\mathrm{BER}}, 1 \leq n \leq n_{\mathrm{T}} \\
\operatorname{Tr}[\mathbf{P}]=n_{\mathrm{T}}
\end{gathered}
$$

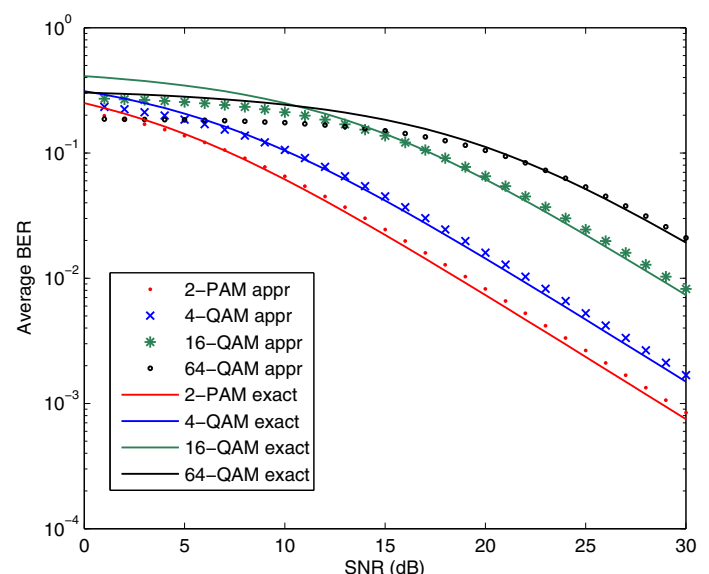

Figure 1. Exact and approximate average BER for uncoded $M$-QAM, $n_{\mathrm{T}}=n_{\mathrm{R}}=3$, i.i.d Rayleigh-faded channel

\section{B. Minimize Power}

$$
\begin{gathered}
\min \operatorname{Tr}[\mathbf{P}] \\
\text { s.t. } \bar{P}_{e, n} \leq \overline{\mathrm{BER}}, 1 \leq n \leq n_{\mathrm{T}} \\
\sum_{n=1}^{n_{\mathrm{T}}} \log _{2} M_{n}=R
\end{gathered}
$$

Problem $A$ can be solved by searching over all matrices $\mathbf{P}$ that satisfy $\operatorname{Tr}[\mathbf{P}]=n_{\mathrm{T}}$, calculating every $\bar{P}_{e, n}$ from Tables II-III and selecting the solution that satisfies $\bar{P}_{e, n} \leq \overline{\mathrm{BER}}, 1 \leq$ $n \leq n_{\mathrm{T}}$, and achieves the maximum sum bit rate.

Problem $B$ can be solved using the inverse of the functions in Tables II-III that give the matrix $\mathbf{P}$ required to satisfy a given average BER per signal for each constellation cardinality. These inverse functions are shown in Tables IV and V for uncoded and coded modulations, respectively.

In both cases efficient bit and power loading algorithms available in the literature can be leveraged (cf. Section IV-C).

Table IV

REQUIRED $\theta$ TO SATISFY $\bar{P}_{e}$ : UNCODED MODULATIONS

\begin{tabular}{|c|c|}
\hline 2-PAM & $\theta\left(\bar{P}_{e}\right)=\left(2 \sqrt{\pi} \bar{P}_{e}\right)^{-1 / \alpha}-1$ \\
\hline 4-QAM & $\theta\left(\bar{P}_{e}\right)=2\left[\left(2 \sqrt{\pi} \bar{P}_{e}\right)^{-1 / \alpha}-1\right]$ \\
\hline$M$-QAM & $\theta\left(\bar{P}_{e}\right)=\frac{2}{3}(M-1)\left[\left(1 / 2 \log _{2}(M) \sqrt{\pi} \bar{P}_{e}\right)^{-1 / \alpha}-1\right]$ \\
\hline
\end{tabular}

Table V

REQUiREd $\theta$ TO SATISFy $\bar{P}_{e}$ : CODED MODULATIONS

\begin{tabular}{|c|c|}
\hline 2-PAM & $\theta\left(\bar{P}_{e}\right)=\frac{2}{d_{\text {free }}}\left[\left(\frac{\bar{P}_{e}}{\beta}\right)^{-1 / \alpha}-1\right]$ \\
\hline 4-QAM & $\theta\left(\bar{P}_{e}\right)=\frac{4}{d_{\text {free }}}\left[\left(\frac{\bar{P}_{e}}{\beta}\right)^{-1 / \alpha}-1\right]$ \\
\hline$M$-QAM & $\theta\left(\bar{P}_{e}\right)=\frac{4(M-1)}{3 d_{\text {free }}}\left[\left(\frac{\bar{P}_{e}}{4 \beta} \log _{2} M\right)^{-1 / \alpha}-1\right]$ \\
\hline
\end{tabular}




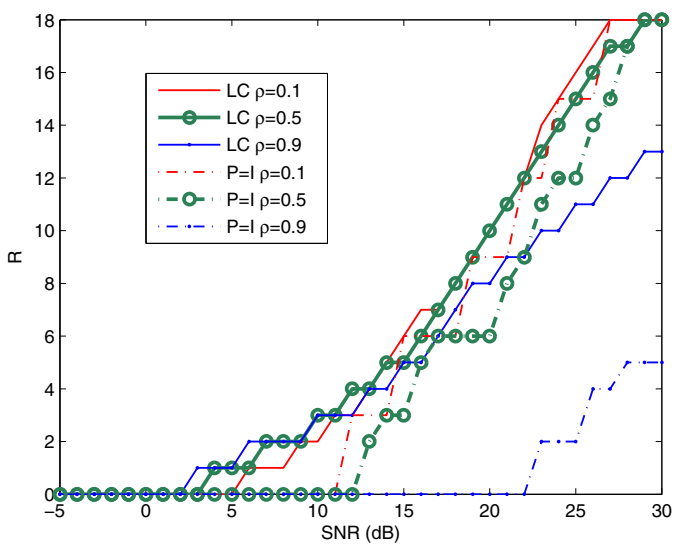

Figure 2. Bit rate vs. SNR, 6 uncoded bit rates, $n_{\mathrm{T}}=3, n_{\mathrm{R}}=4, \overline{\mathrm{BER}}=10^{-2}$.

\section{Levin-Campello Bit Loading}

The Levin-Campello algorithm [2] is known to optimally solve the problem of bit loading with discrete constellations at a target error probability. Its key tenet is that each additional information unit to be transmitted should be allocated to the signaling channel (vector in our case) that requires the least incremental energy for its transport. Incremental energies for the modulation schemes that we are considering can be obtained from Table IV and the application of the algorithm to the solution of Problems $A$ and $B$ is then quite straightforward.

\section{Performance Results}

In order to assess the performance of an optimized system relative to a non-optimized one, we shall consider three possible scenarios in terms of modulation and coding schemes available at the transmitter.

a) Uncoded modulation: $M_{n}$ takes values in the set $\{0$, $2,4,8,16,32,64,256,512\}$. Although cardinalities beyond $M_{n}=64$ might be unpractical, this scenario illustrates what would happen if the modulation cardinality were unbounded.

b) 6 uncoded bit rates: $M_{n}$ takes values in the set $\{0$, $2,4,8,16,32,64\}$. This is a realistic uncoded scenario.

c) 12 bit rates with convolutional coding: $M_{n}$ takes values in the set $\{0,2,4,8,16,32,64\}$. Each of the signals will either be uncoded or coded with a rate- $1 / 2$ convolutional code of generator polynomials $(133,171)$ and $d_{\text {free }}=10$.

\section{A. Bit Rate Maximization}

Figs 2-3 show the achievable bit rate $R$ as function of the SNR when the power allocation $\mathbf{P}$ is optimized in comparison with the case $\mathbf{P}=\mathbf{I}$. Different values for the channel correlation parameter $\rho$ (cf. Eq. 9) are used while $n_{\mathrm{T}}=3$, $n_{\mathrm{R}}=4$ and $\overline{\mathrm{BER}}=10^{-2}$. By optimizing $\mathbf{P}$, higher bit rates are always achieved. The gains in bit rate are larger when $\rho$ is high since, in that case, optimizing $\mathbf{P}$ allows adapting to the fact that the output SINRs become very different. Also, optimizing $\mathbf{P}$ allows extending the range of operational SNR since nonzero bit rates are possible at low SNRs even when transmission is

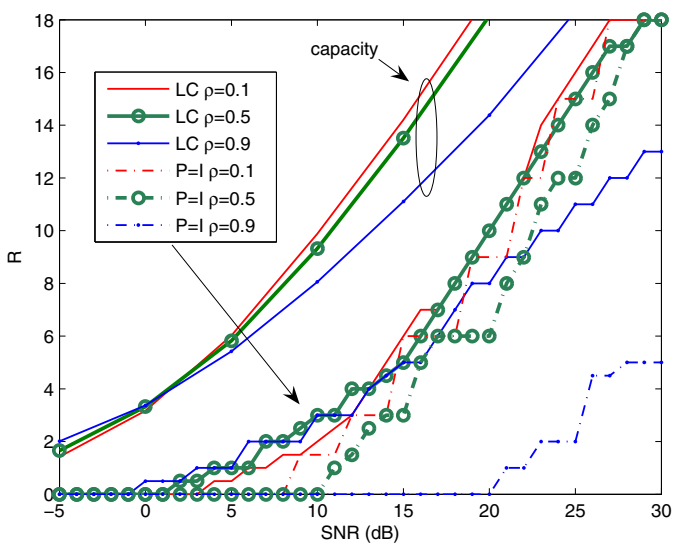

Figure 3. Bit rate vs. SNR, 12 bit rates with convolutional coding, $n_{\mathrm{T}}=3$, $n_{\mathrm{R}}=4, \overline{\mathrm{BER}}=10^{-2}$. Capacity shown as reference.

not possible using simply $\mathbf{P}=\mathbf{I}$. With a richer set of bit rates via coding, this range extension is more pronounced as can be seen by comparing both figures.

It is known that correlation has the following effect on the CDIT capacity (with the optimum transmit covariance): correlation is beneficial at low SNR and it is detrimental above a critical SNR [6]. Figure 3 shows the CDIT capacity according to [1] for the channel model we are considering. Interestingly, correlation has the same effect on the bit rate achieved by the proposed bit loading with ZF receiver, with the particularity that the critical SNR is higher in this case. With simple convolutional codes and a ZF receiver, the proposed scheme is seen to perform within $9 \mathrm{~dB}$ of capacity at $\overline{\mathrm{BER}}=10^{-2}$ and SNR $=20 \mathrm{~dB}$.

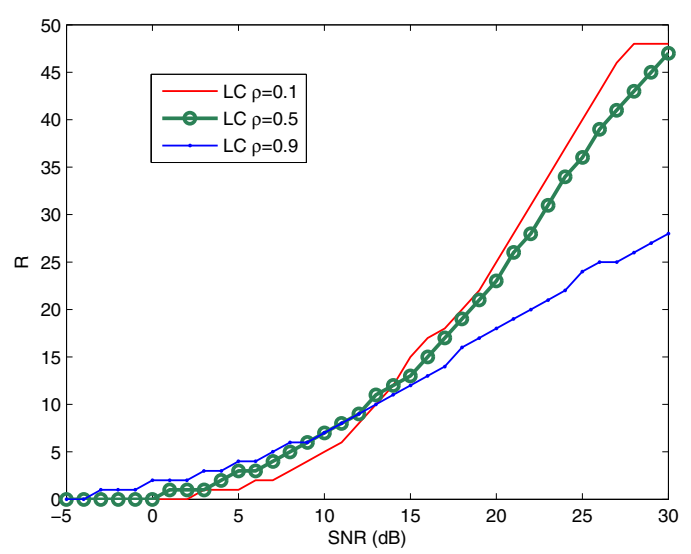

Figure 4. Bit rate vs. SNR, 6 uncoded bit rates, $n_{\mathrm{T}}=8, n_{\mathrm{R}}=10, \overline{\mathrm{BER}}=10^{-2}$.

To illustrate the applicability of the optimization procedure to settings with large numbers of antennas, Fig. 4 presents some achievable bit rates as function of the SNR with 8 transmit and 10 receive antennas and $\overline{\mathrm{BER}}=10^{-2}$. 


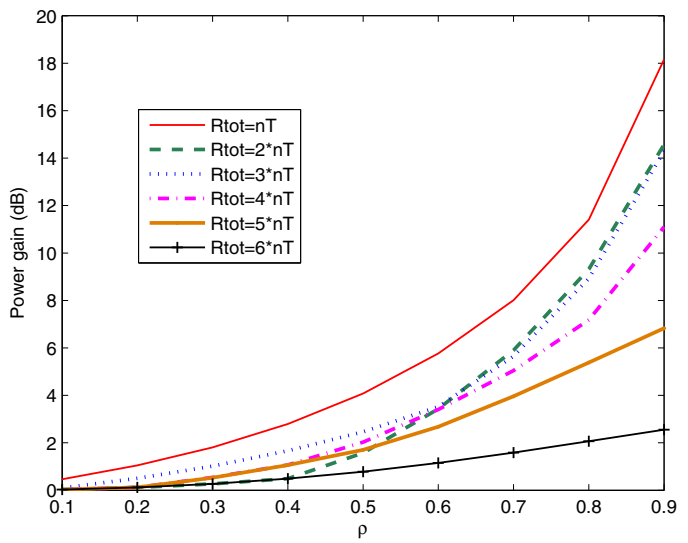

Figure 5. Power gain vs. $\rho, 6$ uncoded bit rates, $n_{\mathrm{T}}=3, n_{\mathrm{R}}=4, \overline{\mathrm{BER}}=10^{-2}$.

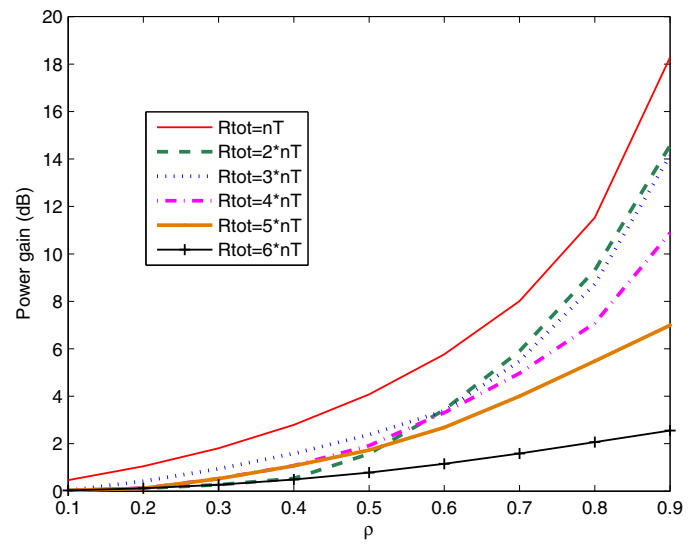

Figure 6. Power gain vs. $\rho, 6$ uncoded bit rates, $n_{\mathrm{T}}=3, n_{\mathrm{R}}=4, \overline{\mathrm{BER}}=10^{-4}$.

\section{B. Power Minimization}

Let us now examine the power gain defined as the $\mathrm{dB}$ difference in $\operatorname{Tr}[\mathbf{P}]$ that results from optimizing $\mathbf{P}$ versus using $\mathbf{P}=\mathbf{I}$. Fig. 5 shows this gain for $n_{\mathrm{T}}=3, n_{\mathrm{R}}=4, \overline{\mathrm{BER}}=10^{-2}$ with 6 uncoded bit rates. Gains in excess of $10 \mathrm{~dB}$ can be obtained for high $\rho$.

In order to show the dependence on $\overline{\mathrm{BER}}$, Fig. 6 plots the same scenario of Fig. 5 except with $\overline{\mathrm{BER}}=10^{-4}$. The impact of $\overline{\mathrm{BER}}$ on the power gain is seen to be negligible.

In Figs 7-8, we explore the extreme cases when the required bit rate is either $R=n_{\mathrm{T}}$ or $R=6 n_{\mathrm{T}}$. This provides insight on the low- and high-SNR behaviors, respectively. Uncoded modulation is used, with $\overline{\mathrm{BER}}=10^{-2}$. Contrasting these figures, we can see that limiting the number of bit rates without coding limits the power gain at high SNR since no highercardinality constellations are available to profit from such high SNR. This effect does not occur at low SNR. Higher gains are achieved for $R=n_{\mathrm{T}}$ (low SNR) than for $R=6 n_{\mathrm{T}}$ (high SNR).

\section{CONCLUSION}

We have developed expressions that relate the channel statistics, the precoding vectors, and the power allocation, with

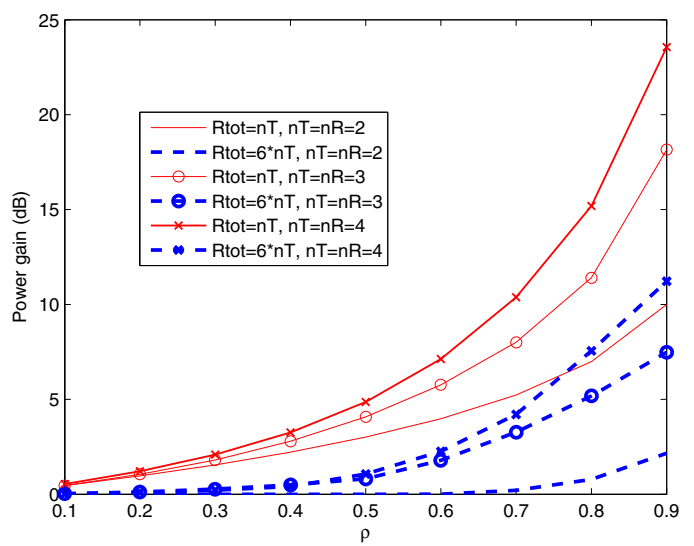

Figure 7. Power gain with uncoded modulation, $\overline{\mathrm{BER}}=10^{-2}$.

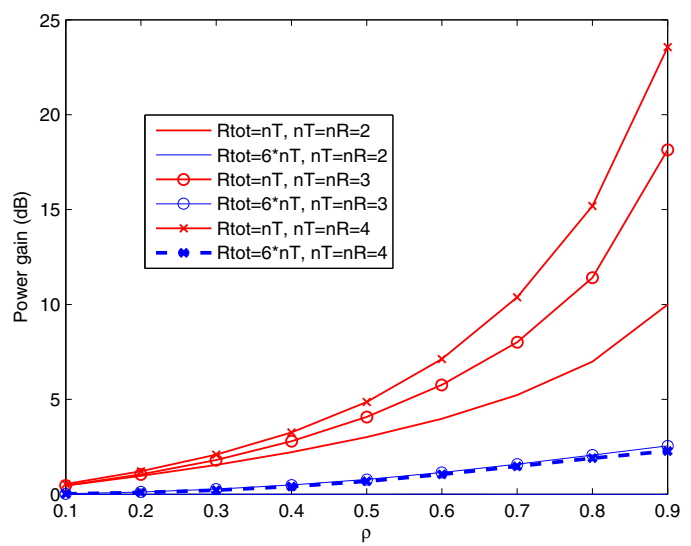

Figure 8. Power gain with 6 uncoded modulations, $\overline{\mathrm{BER}}=10^{-2}$.

the average BER at the output of a $\mathrm{ZF}$ receiver for uncoded or coded $M$-QAM transmission in single-user MIMO. These expressions enable bit and power loading procedures that result in substantially higher bit rates and/or less power consumption. The formulation leverages available loading algorithms to render the optimization process highly efficient.

\section{REFERENCES}

[1] A. M. Tulino, A. Lozano, and S. Verdú, "Capacity-achieving input covariance for single-user multi-antenna channels," IEEE Trans. on Wireless Communications, vol. 5, no. 3, pp. 662-671, March 2006.

[2] J. Campello, "Practical bit loading for DMT," Proc. Int'l Conf. Communications, Vancouver, Canada, vol. 2, pp. 801-805, June 1999.

[3] A. Paulraj, Introduction to Space-Time Wireless Communications. Cambridge University Press, 2003.

[4] P. Li, D. Paul, R. Narasimhan, and J. Cioffi, "On the distribution of SINR for the MMSE MIMO receiver and performance analysis," IEEE Trans. Inform. Theory, vol. 52, no. 1, pp. 271-286, Jan. 2006.

[5] J. Proakis, Digital Communications, 4th ed. McGraw Hill, 2000.

[6] A. M. Tulino, A. Lozano, and S. Verdú, "Impact of Antenna Correlation on the Capacity of Multiantenna Channels," IEEE Trans. on Information Theory, vol. 51, no. 7, pp. 2491-2509, July 2005.

[7] A. Scaglione, G. Giannakis, and S. Barbarossa, "Redundant filterbank precoders and equalizers. I. Unification and optimal designs," IEEE Trans. on Signal Processing, vol. 47, no. 7, pp. 1988-2006, July 1999. 